STRUCTURAL

CHEMISTRY

ISSN 2053-2296

\section{Sweet like chocolate}

\author{
Iain D. H. Oswald*
}

Strathclyde Institute of Pharmacy and Biomedical Sciences, 161 Cathedral Street, Glasgow G4 ORE, Scotland. *Correspondence e-mail: iain.oswald@strath.ac.uk

In the July issue of Acta Crystallographica Section C, Nicholls et al. (2019) report the discovery of a new polymorph of $\alpha \beta$-D-lactose through the oven drying of concentrated aqueous solutions of $\mathrm{D}$-lactose. The authors interest in this system was piqued in relation to the properties of chocolate crumb and the process by which the crumb is made. The drying process by which the crumb is produced is a potential mine of interesting solidstate transformations and in this case the authors simplified the system from a complex mixture of milk and sucrose to a concentrated aqueous solution of lactose. This simplification of the system has enabled the authors to focus in on the phase transitions that occur in the system and analyse the crystallization products with high-quality crystallographic information. Lucky for Acta Crystallographica Section $C$ that such a delicious structural study was published here!

D-Lactose possesses a complex solid-state behaviour due to the potential to form two anomers $(\alpha$ and $\beta$ ). Prior to this study there were two known polymorphs of $\alpha$-lactose (Platteau et al., 2004 2005), one polymorph of the $\beta$-anomer (Hirotsu \& Shimada, 1974), as well as a mixed $\alpha \beta$-form that is composed of both anomers (Guiry et al., 2008). If the phase space was not complex enough, $\alpha$-lactose possesses a hydrated structure (Smith et al., 2005) that is used to access the two anhydrous forms, one of which is more hygroscopic than the other. Prior to the present study, a mixture of methods have previously been used to characterize the materials, with the hydrate and $\beta$-polymorph being characterized by single-crystal diffraction, whilst the $\alpha$-polymorphs were identified through powder X-ray diffraction due to the reconstructive nature of the phase transitions from the hydrate. The fact that any information can be taken from the powder patterns is quite amazing given the complexity of the molecules and the low symmetries involved. In the case of this study, Nicholls et al. employed a mixture of diffraction techniques, as well as theoretical methods, to enable the characterization of the phases of lactose obtained from a range of drying conditions (four different temperatures: 100, 110, 120 and $140{ }^{\circ} \mathrm{C}$ ). Whilst the powder patterns of single phases of low-symmetry systems may be complex enough, Nicholls et al. encountered multiple phases in the dried samples that required careful dissection into the constituent parts. Samples dried at $100{ }^{\circ} \mathrm{C}$ were identified as a mixture of $\beta$-lactose and $\alpha$-lactose monohydrate in varying proportions. For samples dried at temperatures above $100{ }^{\circ} \mathrm{C}$, the authors were able to identify known phases, such as $\beta$-lactose, but this phase only accounted for a small portion of the pattern (23\%). Fortunately for the authors, the crystallization gods were with them on this study and through a thorough microscopic examination of the powder they were able to identify several single crystals of suitable size for single-crystal X-ray diffraction. One of these crystals $(76 \times 24 \times 18 \mu \mathrm{m})$ indexed as a new monoclinic phase that was able to account for $57 \%$ of the diffraction pattern from samples dried at $110{ }^{\circ} \mathrm{C}$; the proportion of this phase increased to approximately $90 \%$ at higher drying temperatures. At $110{ }^{\circ} \mathrm{C}$, the other phases were $\beta$-lactose, $\alpha \beta$-lactose (triclinic form) and a portion of the stable anhydrous form of $\alpha$-lactose. The authors observed that as the drying temperature increased the only other main phase present was the triclinic form of $\alpha \beta$-lactose.

In both the triclinic and newly observed monoclinic structure of $\alpha \beta$-lactose, the molecules pack with alternating layers of the $\alpha$ - and $\beta$-anomers, with very slight differences in the overall packing, giving an r.m.s. deviation of 0.336 using the 'Crystal Packing Similarity' feature in Mercury (Macrae et al., 2008). This similar packing ensures that the energy difference between the two phases is negligible, with no phase being clearly more stable. Dispersion-corrected density functional theory (DFT-D) calculations provided support to the structural analysis and ensured that $\mathrm{H}$-atom positions were refined in the 


\section{scientific commentaries}

correct place. In this specific case, theory was able to help identify previously overlooked hydrogen bonding that was restrained in the final single-crystal study.

The study of Nicholls et al. is a lesson in the power of observation and the rigorous analysis of powder samples. Without the microscopic analysis of the powder and the sequential identification of the single crystals, the new phase would have required an even greater effort on the part of the authors to solve and identify. The identification of multiple phases in this study (up to four phases) is an example where the full fitting of diffraction patterns to the known phases, however complex, is an essential practice so that detailed structural information can be gained and utilized in further studies of these ubiquitous materials.

\section{References}

Guiry, K. P., Coles, S. J., Moynihan, H. A. \& Lawrence, S. E. (2008). Cryst. Growth Des. 8, 3927-3934.

Hirotsu, K. \& Shimada, A. (1974). Bull. Chem. Soc. Jpn, 47, 1872 1879.

Macrae, C. F., Bruno, I. J., Chisholm, J. A., Edgington, P. R., McCabe, P., Pidcock, E., Rodriguez-Monge, L., Taylor, R., van de Streek, J. \& Wood, P. A. (2008). J. Appl. Cryst. 41, 466-470.

Nicholls, D., Elleman, C., Shankland, N. \& Shankland, K. (2019). Acta Cryst. C75, 904-909.

Platteau, C., Lefebvre, J., Affouard, F. \& Derollez, P. (2004). Acta Cryst. B60, 453-460.

Platteau, C., Lefebvre, J., Affouard, F., Willart, J.-F., Derollez, P. \& Mallet, F. (2005). Acta Cryst. B61, 185-191.

Smith, J. H., Dann, S. E., Elsegood, M. R. J., Dale, S. H. \& Blatchford, C. G. (2005). Acta Cryst. E61, o2499-o2501. 